\title{
Физические основы самосогласованного моделирования процессов генерации интерфейсных состояний и транспорта горячих носителей в транзисторах на базе структур металл-диэлектрик-кремний
}

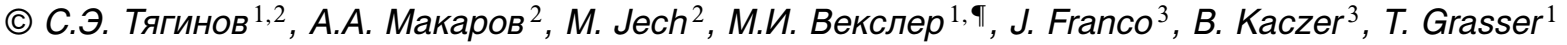 \\ ${ }^{1}$ Физико-технический институт им. А.Ф. Иоффее Российской академии наук, \\ 194021 Санкт-Петербург, Россия \\ 2 TU Vienna, Institute for Microelectronics, \\ Vienna 1040, Austria \\ ${ }^{3}$ IMEC, \\ Kapeldreef 75, 3001 Leuven, Belgium \\ ฯ E-mail: Vexler@mail.ioffe.ru
}

(Получена 22 июня 2017 г. Принята к печати 23 июня 2017 г.)

\begin{abstract}
Впервые выполнено детальное моделирование деградации, вызываемой горячими носителями, основанное на самосогласованном рассмотрении транспорта носителей и генерации дефектов на границе раздела $\mathrm{SiO}_{2} / \mathrm{Si}$. Данная модель апробирована с использованием деградационных данных, полученных в декананометровых $n$-канальных полевых транзисторах. Показано, что взаимное влияние двух указанных аспектов велико и их независимое моделирование влечет серьезные количественные ошибки. При вычислении функций распределения носителей по энергии учитывалась реальная зонная структура кремния и такие механизмы, как ударная ионизация, рассеяние на ионизованной примеси, а также электрон-фононные и электронэлектронные взаимодействия. На микроскопическом уровне генерация дефектов рассматривалась как суперпозиция одночастичного и многочастичного механизмов разрыва связи $\mathrm{Si}-\mathrm{H}$. Очень важным прикладным аспектом данной работы является тот факт, что наша модель позволяет надежно оценивать ресурс работы транзистора, подверженного воздействию горячих носителей.
\end{abstract}

DOI: $10.21883 /$ FTP.2018.02.45452.8652

\section{1. Введение}

На границе раздела диэлектрик/кремний полевого МДП транзистора (МДП - металл-диэлектрик-полупроводник) всегда присутствуют поверхностные состояния, которые могут формировать заряженные дефекты. Присутствие подобных дефектов приводит к локальным искажениям электростатики прибора, что проявляется, например, в сдвиге порогового напряжения полевого транзистора. Они также влияют на перенос электронов в приграничном индуцированном канале.

Поверхностная концентрация ловушек $\left(N_{i t}\right)$ может меняться со временем в зависимости от условий использования транзистора. При этом эволюция плотности $N_{i t}$ определяет эволюцию (деградацию) характеристик прибора. Обычно в экспериментах по деградации используются более высокие (по сравнению с рабочими) напряжения и температуры. Основная прикладная задача заключается в прогнозировании ресурса прибора именно в рабочем режиме. Проблема состоит в том, что при переходе от „жестких“ условий воздействия на прибор к более „мягким“ рабочим напряжениям физические механизмы, ответственные за разрушение транзистора, могут полностью измениться [1-3], тем самым делая взятую за основу феноменологическую/эмпирическую модель несостоятельной. Поэтому для адекватного моделирования процессов деградации необходимо понимание и точное описа- ние физических механизмов, соответствующих реальному режиму.

В ежегодно публикуемых прогнозах развития электронной промышленности ITRS (International Technology Roadmap for Semiconductors) задача надежности функционирования полупроводниковых приборов декларируется как проблема первостепенной важности [4]. При этом основной режим повреждения транзисторов новых поколений связан с деградацией подзатворного диэлектрика, вызываемой горячими носителями. Это подтверждается, скажем, недавними исследованиями компании Intel $[5,6]$.

Настоящая работа посвящена подробному физическому моделированию процессов формирования интерфейсных ловушек в МДП структуре, выполняемому совместно с моделированием изменений кинетики горячих электронов в такой структуре. Мы сначала представим модель генерации дефекта в транзисторах на основе системы диоксид кремния/кремний $\left(\mathrm{SiO}_{2} / \mathrm{Si}\right)$. Затем будут приведены результаты расчетов плотности ловушек $N_{i t}$ для различных условий воздействия на прибор. На основании этих данных будет сделан вывод о необходимости самосогласованного расчета плотности энергетического распределения электронов и дефектов. Интерфейс диэлектрик/кремний рассматривается как двумерный объект, причем считается достаточным анализировать изменения только вдоль координаты сток-исток. Соответственно плотность $N_{i t}$ становится функцией латеральной координаты $x$ и времени $t$, те. $N_{i t}=N_{i t}(x, t)$. 


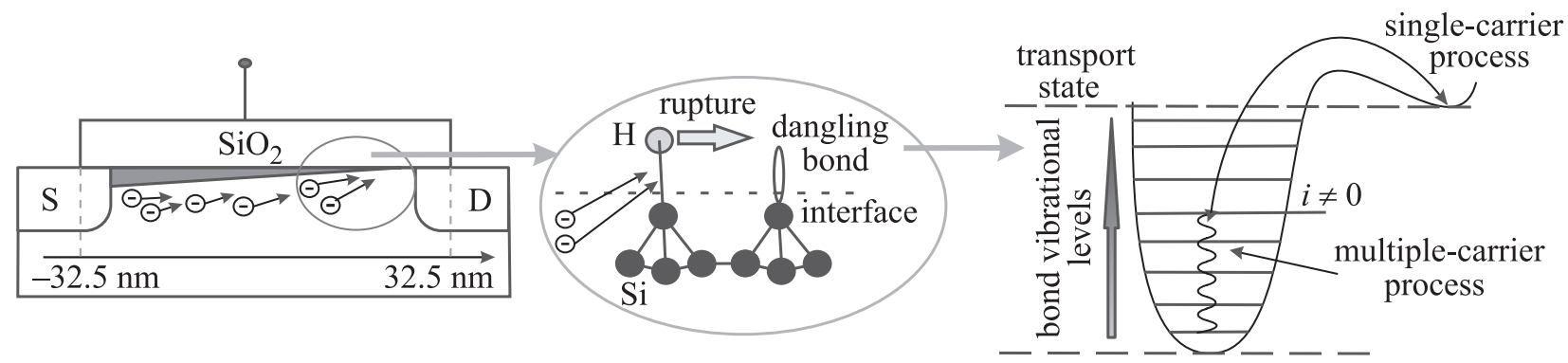

Рис. 1. Схематическое изображение механизма генерации дефектов в ходе деградации, вызываемой горячими носителями. $\mathrm{S}$ - исток, D - сток.

\section{2. Модель формирования интерфейсного дефекта в ходе деградации, вызываемой горячими носителями}

\section{1. Возможная схема возникновения дефекта}

Как известно, граница раздела $\mathrm{SiO}_{2} / \mathrm{Si}$ является неупорядоченной системой [7]. Среди прочего это проявляется в наличии оборванных связей $\mathrm{Si}-$. Данные связи могут захватывать носители заряда, образуя таким образом заряженные дефекты, называемые $P_{b}$-центрами. Для пассивации свободных связей $\mathrm{Si}-$ применяют водород $(\mathrm{H})$, который внедряется непосредственно при выращивании диэлектрической пленки. При участии водорода формируются пассивные связи $\mathrm{Si}-\mathrm{H}$. Эти связи, однако, могут быть разорваны при бомбардировке интерфейса горячими носителями, что и составляет суть деградации [8-10]. Поверхностная плотность пассивных связей $\mathrm{Si}-\mathrm{H}\left(N_{0}\right)$ лимитирует концентрацию $N_{i t}$. Величина $N_{0}$ определяется конкретным технологическим процессом; как правило, это значение лежит в диапазоне $(5-7) \cdot 10^{13} \mathrm{~cm}^{-2}$.

Разрыв связи происходит благодаря комбинированному действию двух процессов - одночастичного и многочастичного [2,9-11]. Первый из них запускается одиночным горячим носителем, который может доставить к интерфейсу энергию, равную энергии разрыва связи $(2.6$ эВ [12]) или выше. Данный механизм является доминантным в приборах с высокими напряжениями стресса/функционирования и соответствует „классической“ деградации, вызываемой горячими носителями. Однако в декананометровых полевых транзисторах (с низкими рабочими напряжениями) концентрация горячих носителей низкая, поэтому доминантными становятся многочастичные механизмы $[2,11]$. При этом бомбардировка производится несколькими „холодными“ частицами, которые постепенно возбуждают связь, что в конечно итоге приводит к ее диссоциации. Для точного описания реакции разрыва связи необходимо рассматривать все возможные суперпозиции этих механизмов [13-16], что и было сделано в нашей модели деградации, вызываемой горячими носителями $[15,17]$. Модель также учитывает взаимодействие локального электрического поля $F(x, t)$ с дипольным моментом связи $\mathrm{Si}-\mathrm{H}[14,15]$. Такое взаимодействие приводит к уменьшению энергии разрыва связи. Из-за неупорядоченности интерфейса $\mathrm{SiO}_{2} / \mathrm{Si}$ эта энергия является флуктуирующей величиной и описывается нормальным распределением, что также учитывается в модели $[14,15]$.

\section{2. Роль энергетического распределения электронов}

Для моделирования темпов обоих механизмов следует „различать“ горячие и холодные носители [18]. Эта информация содержится в обобщенной функции распределения (ФР) носителей по энергии $f(E) \rho(E)=d n(E, x) / d E\left({ }^{э} \mathrm{~B}^{-1} \cdot \mathrm{cm}^{-3}\right)$. Обобщенная $\Phi \mathrm{P}$ является произведением вероятности занятия состояния с данной энергией $f(E)$ на плотность состояний $\rho(E)$. Наша модель использует симулятор ViennaSHE [17], ocyществляющий детерминистическое решение уравнения Больцмана. Для каждой точки $x$ интерфейса ViennaSHE вычисляет ФР для заданных архитектуры прибора и напряжений.

Функции распределения затем используются для моделирования темпа $\left(\mathrm{c}^{-1}\right)$ диссоциации связей горячими носителями $[14,15]$ :

$$
I\left(E_{a}\right)=\int f(E) \rho(E) \sigma\left(E, E_{a}, d, F\right) v(E) d E+I_{\text {th }},
$$

где $\sigma$ - эффективное сечение рассеяния одночастичного/многочастичного процесса, $v(E)$ - групповая скорость носителей. На величину $\sigma$ помимо энергии $E$ влияют энергия связи $E_{a}$, ее дипольный момент $d$ и значение поля $F$. Второе слагаемое в (1) представляет собой темп термоактивации разрыва связи. Интегрирование осуществляется по всему энергетическому спектру.

При возбуждении связи многочастичным процессом на уровень $i$ (см. рис. 1) энергия, необходимая для диссоциации, эффективно уменьшается на величину энергетического положения этого уровня $E_{i}$. Это учитывается 
при вычислении темпа диссоциации возбужденной связи:

$I_{i}\left(E_{a}\right)=\int f(E) \rho(E) \sigma\left(E_{\mathrm{eff}, i}\right) v(E) d E+\omega_{t h} \exp \left(-\frac{E_{\mathrm{eff}, i}}{k T}\right)$.

Функция $\sigma\left(E_{\mathrm{eff}, i}\right)$ отлична от нуля при положительном значении энергии $E_{\mathrm{eff}, i}$, которая вычисляется как $E_{\mathrm{eff}, i}=E-E_{a}+E_{i}+d F$, где член $d F$ отражает вклад взаимодействия дипольного момента связи с полем. Через $\omega_{t h}$ обозначена „частота попыток“ термического разрыва, $k$ - постоянная Больцмана, $T$ - температура решетки. Результирующий темп разрыва связей моделируется как суперпозиция вкладов всех уровней, т. е.

$$
I\left(E_{a}\right)=\sum I_{i}\left(E_{a}\right) .
$$

Энергия $E_{a}$ является флуктуирующей величиной, что также следует учитывать при нахождении темпов разрыва связей:

$$
I=\int I\left(E_{a}\right) \Gamma\left(E_{a},\left\langle E_{a}\right\rangle, \delta_{a}\right) d E_{a},
$$

где $\left\langle E_{a}\right\rangle$ - среднее значение энергии разрыва, $\delta_{a}-$ ее среднеквадратичное отклонение, а $\Gamma\left(E_{a},\left\langle E_{a}\right\rangle, \delta_{a}\right)-$ плотность нормального распределения.

Встраиваемые дефекты влияют на электростатику прибора, изменяя профиль поля $F(x, t)$ и потенциала в структуре. Одновременно происходит снижение подвижности носителей. Как следствие, форма ФР, фигурирующей в (1), также искажается. Поэтому для точного моделирования деградации, вызываемой горячими носителями, требуется проводить самосогласованный расчет темпов генерации дефектов и функций распределения носителей по энергии.

\section{3. Результаты расчетов в рамках модели и обсуждение}

Самосогласованное моделирование транспорта носителей и кинетики генерации дефектов является весьма ресурсоемкой задачей, поэтому ранее в нашей модели набор ФР для разных координат $x$ вычислялся только один раз для $t=0 \quad[14,15,19]$. В настоящей работе делается шаг вперед: оценивается влияние встраиваемых дефектов на функции распределения носителей. Оценки проводятся для декананометровых транзисторов с длиной затвора 65 нм и оксинитридом кремния $(\mathrm{SiON})$ в качестве подзатворного диэлектрика (физическая толщина слоя $2.5 \mathrm{Hм})$.

\section{1. Программные средства моделирования}

Используемая нами программа-симулятор ViennaSHE для нахождения ФР осуществляет их разложение в ряд сферических гармоник [17]. Симулятор включает эффекты реальной зонной структуры $\mathrm{Si}$ для электронов/дырок вплоть до высоких энергий. ViennaSHE учитывает такие механизмы рассеяния, как ударная ионизация, рассеяние на заряженной примеси, поверхностное рассеяние, электрон-фононное и электрон-электронное взаимодействия. Последний тип взаимодействия оказывается основным фактором деградации, вызываемой горячими носителями, в транзисторах с длиной канала $<120$ HM $[20,21]$.

\section{2. Предварительная оценка масштаба эффекта}

В целях предварительной оценки возможного масштаба влияния дефектов на ФР был выполнен сравнительно простой расчет для заданного постоянного значения $N_{i t}=5 \cdot 10^{12} \mathrm{~cm}^{-2}$, как если бы дефекты с заданной плотностью были равномерно распределены вдоль интерфейса. Это, однако, сугубо искусственный пример: на практике деградация, вызываемая горячими носителями, является сильно неоднородным явлением и профили $N_{i t}$ имеют выраженный пик около точки отсечки транзистора $[8,9,22]$.

На рис. 2 показана серия ФР, вычисленных при $V_{d s}=V_{g s}=1.8 \mathrm{~B}\left(V_{d s}, V_{g s}\right.$ - напряжения сток-исток и затвор-исток соответственно) и температуре $T=25^{\circ} \mathrm{C}$. Видно, что встраивание интерфейсных состояний значительно изменяет форму распределений, особенно „населенность“" их высокоэнергетичных хвостов. Эффект наиболее значим для областей ближе к стоку: например, при латеральных координатах $x=15.0$ и 18.5 нм получается уменьшение чисел заполнения в 2-3 раза. Из рис. 2 можно заключить, что наличие вполне обычных концентраций дефектов серьезно изменяет ФР.

\section{3. Процедура количественного расчета}

Для точной оценки степени влияния встраивания дефектов на транспорт носителей, как уже отмечалось, необходимо самосогласованное описание этих процессов. При этом для вычисления ФР в определенной точке у интерфейса с координатой $x$ нужно располагать значением $N_{i t}$ не только в данной точке, но и во всех точках на интерфейсе.

Общий алгоритм выглядит следующим образом. В начальный момент считается, что $N_{i t}(x, 0)=0$. Для любого момента $t$ сначала рассчитывается распределение потенциала и электрического поля $F(x, t)$ посредством решения уравнения Пуассона. Это позволяет вычислить ФР для различных координат в заданный момент времени. Располагая ФР, мы находим темп генерации дефектов $I$ (см. формулы $(1)-(4))$, который при умножении на $N_{0}$ и на шаг по времени даст величину новой плотности ловушек: $N_{i t}(x, t+\Delta t)=N_{i t}(x, t)+N_{0} I(x, t) \Delta t$. Эта процедура повторяется циклически с использованием для следующего момента времени уже скорректированного значения $N_{i t}$. 

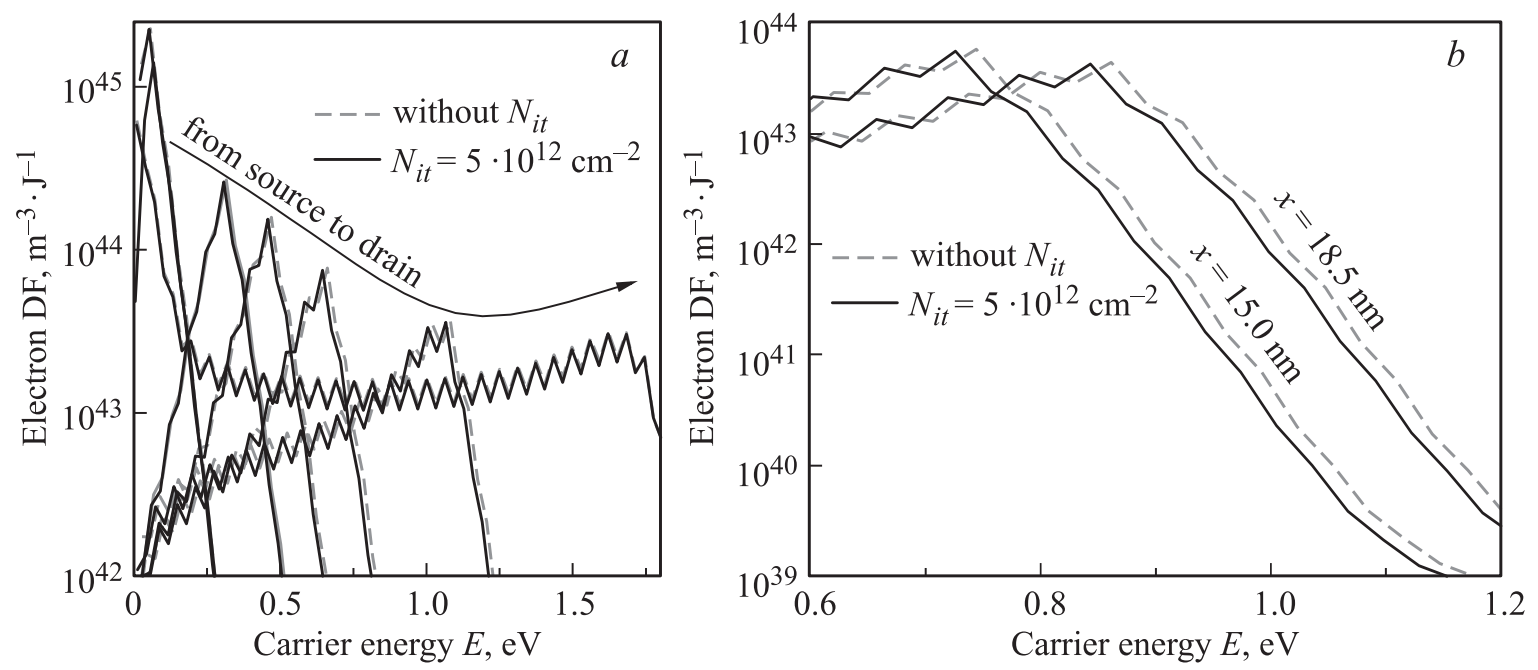

Рис. 2. Серия функций распределения электронов (DF) по энергии $E$, вычисленных с учетом и без учета эффекта $N_{i t}$. $V_{d s}=V_{g s}=1.8 \mathrm{~B}, T=25^{\circ} \mathrm{C} . a-$ эволюция обоих семейств с латеральной координатой. $b-\Phi \mathrm{P}$ для тех позиций $(x=15,18.5$ нм $)$ и энергий, где эффект наиболее значим. Истоку соответствует $x=-32.5$ нм, стоку $-x=32.5$ нм.
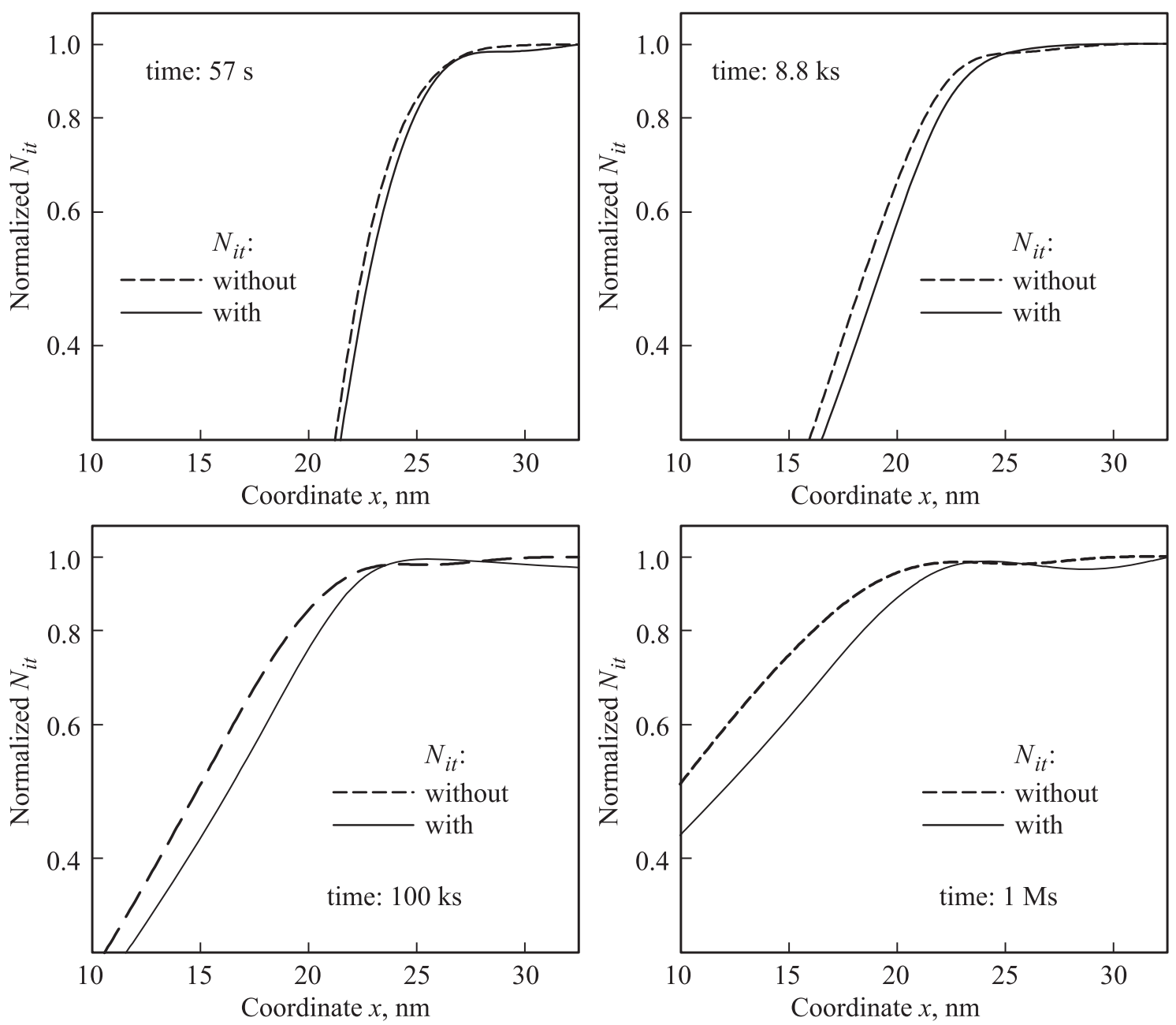

Рис. 3. Профили распределения концентрации ловушек на интерфейсе, $N_{i t}(x)$, вычисленные с учетом и без учета влияния встраиваемых дефектов на транспорт носителей в транзисторе. Показаны только значения $N_{i t}$ в районе максимума, около точки отсечки полевого транзистора. 


\section{4. Примеры точной оценки влияния дефектов}

Приведем примеры, иллюстрирующие влияние дефектов в рамках описанного выше алгоритма с учетом самосогласования. Для сравнения (без самосогласования) мы используем результаты расчета, при котором рассчитываем ФР на всех шагах по времени для нулевого значения $N_{i t}$, т.е. с игнорированием факта эволюции плотности дефектов.

На рис. 3 представлены рассчитанные профили величины $N_{i t}(x, t) / N_{0}$ для четырех моментов времени (показана только область ближе к стоку, где эффекты горячих электронов наиболее сильны). Мы видим различия результатов, которые становятся более заметными со временем. Изменение $N_{i t}(x, t)$ происходит за счет изменений со временем функции распределения электронов и напряженности электрического поля $F(x, t)$. При этом в варианте без самосогласования вычисление ФР происходит неточно, что и обусловливает несоответствие.

На рис. 4 показаны два семейства ФР, которые были получены для тех же латеральных координат, что и на рис. 2, для различных значений времени деградации $\left(t=0\right.$ соответствует „свежему“ прибору с $N_{i t}=0$, т. е. отсутствию самосогласования). Видно, что благодаря влиянию нарастающей $N_{i t}$ населенность может значительно изменяться, особенно при больших временах деградации. Это свидетельствует о том, что данный эффект не может игнорироваться и самосогласованное описание процесса генерации дефектов и транспорта носителей необходимо.

\section{5. Соответствие экспериментальным данным}

Сопоставление экспериментальных и расчетных данных напрямую неосуществимо, так как функции распределения не могут быть непосредственно измерены. Однако имеется возможность подтвердить состоятельность модели путем сравнения эволюции тока стока полевого транзистора, подверженного воздействию горячих носителей.

В качестве метрики деградации используется относительное изменение линейного тока стока $\Delta I_{d, \operatorname{lin}}(t)=$ $=\left(I_{d, \operatorname{lin}}(t)-I_{d, \operatorname{lin} 0}\right) / I_{d, \operatorname{lin} 0}$, где $t-$ время деградации, а $I_{d, \operatorname{lin} 0}$ - ток стока не поврежденного воздействию горячих носителей транзистора. Ток линейного режима соответствует напряжениям сток-исток и затвор-исток$V_{d s}=0.05 \mathrm{~B}$ и $V_{g s}=1.5 \mathrm{~B}$ соответственно. На рис. 5 представлены зависимости $\Delta I_{d, \operatorname{lin} 0}(t)$ для двух условий: $V_{d s}=V_{g s}=1.8$ и $2.0 \mathrm{~B}$ (температура $25^{\circ} \mathrm{C}$ ). Расчетные зависимости $\Delta I_{d, \operatorname{lin}}(t)$ были получены с учетом влияния $N_{i t}$ на функции распределения электронов. Необходимо, однако, подчеркнуть, что достижение возможно точного соответствия токов стока не было задачей работы. Целью статьи являлась оценка масштаба влияния нарастающей концентрации $N_{i t}$ на функции распределения и, как следствие, на деградационные характеристики

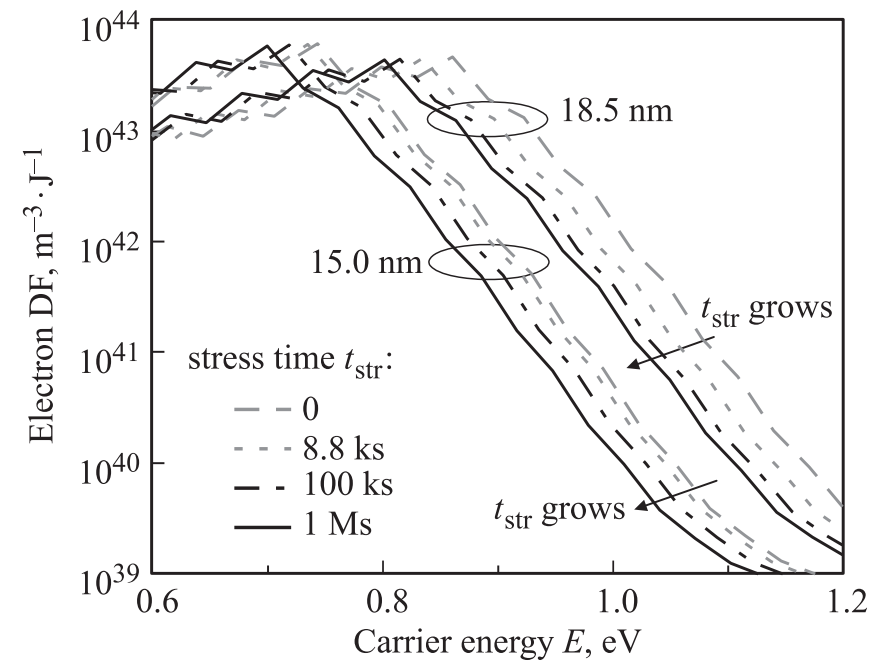

Рис. 4. Семейство электронных функций распределения (DF), рассчитанных с учетом и без учета влияния реальных профилей поверхностной концентрации дефектов $N_{i t}$ для нескольких шагов по времени (stress time $t_{\mathrm{str}}$ ). Приведены ФР для тех значений латеральной координаты, где эффект наиболее сильный. $V_{d s}=V_{g s}=1.8 \mathrm{~B}, T=25^{\circ} \mathrm{C}$.

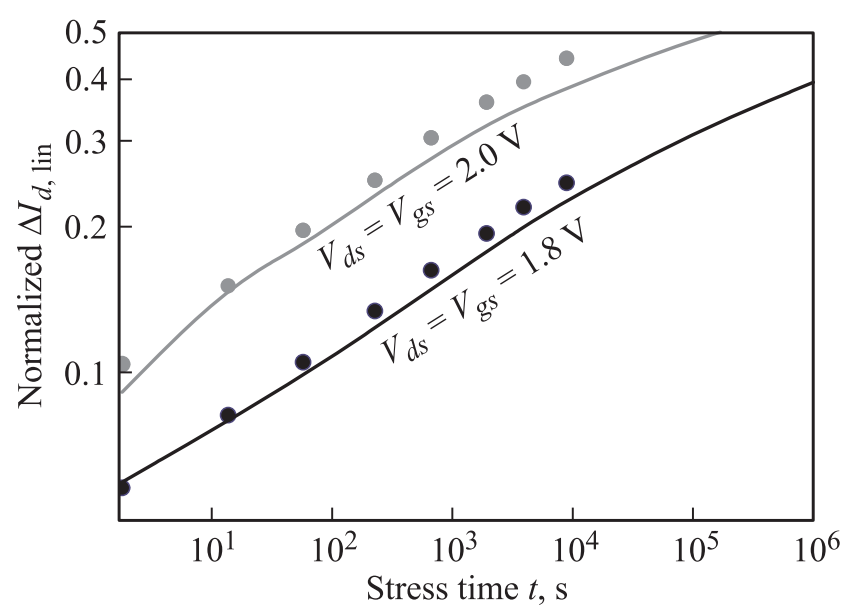

Рис. 5. Относительные изменения тока стока (линейный режим) со временем, $\Delta I_{d, \text { lin }}(t)$, в ходе деградации, вызываемой горячими носителями, для двух условий стресса. Точки эксперимент, сплошные линии - модельный расчет с учетом влияния $N_{i t}$.

(рис. 2-4). Основной же вывод из рис. 5 состоит в том, что используемая модель не приводит к грубым несоответствиям эксперименту в представленных случаях, и это свидетельствует об адекватности нашего подхода и теоретических результатов, показанных на предыдущих рисунках.

\section{4. Заключение}

Мы проанализировали влияние генерации интерфейсных состояний на функции энергетического распределе- 
ния электронов в ходе деградации полевого транзистора, вызываемой горячими носителями. Анализ был выполнен с использованием нашей деградационной модели, рассматривающей одночастичные и многочастичные механизмы разрыва связи кремний-водород, взаимодействие поля в диэлектрике с дипольным моментом связи, а также статистический разброс энергии связи. Вычисления темпов диссоциации основывались на моделировании транспорта носителей с учетом реальной зонной структуры кремния и различных механизмов рассеяния (включая электрон-электронные взаимодействия).

Было показано, что точность вычисления ФР, используемых при анализе процесса генерации дефектов, может значительно влиять и на предсказываемый профиль концентрации ловушек на границе раздела $\mathrm{SiO}_{2} / \mathrm{Si}$, и на такие характеристики прибора, как линейный ток истока. В свою очередь роль ловушек как фактора, определяющего вид функций распределения электронов, увеличивается со временем. В целом результаты показывают, насколько важно взаимное влияние транспорта носителей и формирования дефектов. Для корректного описания и моделирования данного типа деградации требуется самосогласованное рассмотрение этих двух аспектов.

\section{Список литературы}

[1] W. McMahon, A. Haggag, K. Hess. IEEE Trans. Nanotech., 2 (1), 33 (2003).

[2] A. Bravaix, C. Guerin, V. Huard, D. Roy, J. Roux, E. Vincent. In: Proc. Intern. Reliability Physics Symp. (IRPS) (2009) p. 531.

[3] K. Hess, L.F. Register, B. Tuttle, J. Lyding, I.C. Kizilyalli. Physica E, 3, 1 (1998).

[4] International Technology Roadmap for Semiconductors (ITRS) Ch. 5: More Moore (2015).

[5] S. Novak, C. Parker, D. Becher, M. Liu, M. Agostinelli, M. Chahal, P. Packan, P. Nayak, S. Ramey, S. Natarajan. In: 2015 IEEE Intern. Reliability Physics Symp. (2015) p. 2F.2.1.

[6] S. Ramey, Y. Lu, I. Meric, S. Mudanai, S. Novak, C. Prasad, J. Hicks. In: 2015 IEEE Intern. Integrated Reliability Workshop (IIRW) (2015) p. 56.

[7] C.R. Helms, E.H. Poindexter. Rep. Progr. Phys., 57 (8), 791 (1994).

[8] A. Bravaix V. Huard. In: Proc. Europ. Symp. Reliability of Electron Devices Failure Physics and Analysis (ESREF), tutorial (2010) p. 1267.

[9] S. Rauch, G.L. Rosa. In: Proc. Intern. Reliability Physics Symp. (IRPS), tutorial (2010).

[10] S. Tyaginov, T. Grasser. In: Proc. Intern. Integrated Reliability Workshop (IIRW) (2012) p. 206.

[11] W. McMahon, K. Hess. J. Comput. Electron., 1 (3), 395 (2002).

[12] K.L. Brower. Phys. Rev. B, 42 (6), 3444 (1990).

[13] S.E. Tyaginov, I.A. Starkov, O. Triebl, J. Cervenka, C. Jungemann, S. Carniello, J.M. Park, H. Enichlmair, M. Karner, C. Kernstock, E. Seebacher, R. Minixhofer, H. Ceric, T. Grasser. In: Proc. Intern. Symp. the Physical \& Failure Analysis of Integrated Circuits (IPFA) (2010).
[14] M. Bina, S. Tyaginov, J. Franco, Y. Wimmer, D. Osinstev, B. Kaczer, T. Grasser et al. IEEE Trans. Electron Dev., 61 (9), 3103 (2014).

[15] S. Tyaginov, M. Jech, J. Franco, P. Sharma, B. Kaczer, T. Grasser. IEEE Electron Device Lett., 37 (1), 84 (2016).

[16] P. Sharma, S. Tyaginov, M. Jech, Y. Wimmer, F. Rudolf, H. Enichlmair, J.-M. Park, H. Ceric, T. Grasser. Solid-State Electron., 115, pt B, 185 (2016).

[17] M. Bina, K. Rupp, S. Tyaginov, O. Triebl, T. Grasser. In: Proc. Intern. Electron Devices Meeting (IEDM) (2012) p. 713.

[18] S. Tyaginov, I. Starkov, C. Jungemann, H. Enichlmair, J.M. Park, T. Grasser. In: Proc. Europ. Solid-State Device Research Conf. (ESSDERC) (2011) p. 151.

[19] S. Tyaginov, I. Starkov, O. Triebl, H. Enichlmair, C. Jungemann, J.M. Park, H. Ceric, T. Grasser. In: Proc. Intern. Conf. on Simulation of Semiconductor Processes and Devices (SISPAD) (2011) p. 123.

[20] S.E. Rauch, F.J. Guarin, G. La Rosa. IEEE Electron Dev. Lett., 19 (12), 463 (1998).

[21] P. Sharma, S. Tyaginov, S.E. Rauch, J. Franco, A. Makarov, M.I. Vexler, B. Kaczer, T. Grasser. IEEE Electron Dev. Lett., 38 (2), 160 (2017).

[22] S. Tyaginov, I. Starkov, H. Enichlmair, J.M. Park, C. Jungemann, T. Grasser. ECS Trans., 35 (4), 321 (2011).

Редактор Л.В. Шаронова

\section{Physical principles of self-consistent modeling interface state generation and hot carrier transport in metal-oxide-semiconductor field-effect transistors}

\author{
S.E. Tyaginov ${ }^{\mathbf{1}, 2}$, A.A. Makarov², M. Jech², \\ M.I. Vexler ${ }^{1}$, J. Franco ${ }^{3}$, B. Kaczer ${ }^{3}$, T. Grasser ${ }^{1}$ \\ ${ }^{1}$ loffe Institute, \\ 194021 St. Petersburg, Russia \\ 2 TU Vienna, Institute for Microelectronics, \\ Vienna 1040, Austria \\ 3 IMEC, \\ Kapeldreef 75, 3001 Leuven, Belgium
}

\begin{abstract}
For the first time we perform modeling of hotcarrier degradation based on thorough self-consistent consideration of carrier transport and generation of defects at the $\mathrm{SiO}_{2} / \mathrm{Si}$ interface. This approach has been verified against hot-carrier degradation data measured in $n$-channel decananometer field-effect transistors. We have shown that the mutual effect of these two aspects is prominent, thereby suggesting that their independent consideration leads to spurious results. For calculations of carrier energy distribution functions we considered the full-band effects and such energy exchange mechanisms as impact ionization, scattering by ionized impurities, as well as electron-phonon and electron-electron interactions. At the microscopic level, the defect generation was modeled as a superposition of single- and multiplecarrier mechanisms of $\mathrm{Si}-\mathrm{H}$ bond-breakage. A very important applied aspect of this work is that our model allows one to thoroughly estimate the life-time of a transistor subjected to hotcarrier stress.
\end{abstract}

